OPEN ACCESS

Edited by:

Florian Karreth,

Moffitt Cancer Center, United States

Reviewed by:

Monika Wolkers,

Sanquin Diagnostic

Services, Netherlands

Gopal Pandi,

Madurai Kamaraj University, India

*Correspondence:

Myriam Gorospe

myriam-gorospe@nih.gov

Specialty section:

This article was submitted to

Protein and RNA Networks,

a section of the journal

Frontiers in Molecular Biosciences

Received: 08 April 2020

Accepted: 08 May 2020

Published: 26 June 2020

Citation:

Perez de Acha O, Rossi M and Gorospe M (2020) Circular RNAs in Blood Malignancies.

Front. Mol. Biosci. 7:109.

doi: 10.3389/fmolb.2020.00109

\section{Circular RNAs in Blood Malignancies}

\author{
Olivia Perez de Acha, Martina Rossi and Myriam Gorospe* \\ Laboratory of Genetics and Genomics, National Institute on Aging Intramural Research Program, National Institutes of \\ Health, Baltimore, MD, United States
}

Circular (circ)RNAs influence a wide range of biological processes at least in part by interacting with proteins and microRNAs. CircRNAs expressed in the hematopoietic compartment have been increasingly recognized as modulators of physiological and pathological features of hematopoetic stem cell (HSC)-derived populations. In particular, several circRNAs were found to enhance or suppress tumor progression in blood malignancies such as leukemias and lymphomas. Moreover, numerous circRNAs have been proposed to help confer resistance to the conventional treatments used in hematopoietic cancers. Here, we review the most important circRNAs described thus far in acute myeloid leukemia (AML), chronic myeloid leukemia (CML), acute lymphoblastic leukemia (ALL), chronic lymphocytic leukemia (CLL), lymphomas, and multiple myeloma (MM). We discuss the usefulness of circRNAs as diagnostic and prognostic markers and their potential value as therapeutic targets.

Keywords: circular RNAs, blood malignancies, leukemias, lymphomas, non-coding RNA

\section{INTRODUCTION}

The ENCyclopedia Of DNA Elements (ENCODE) project revealed that only 2\% of the human genome encodes proteins (ENCODE Project Consortium, 2012). Subsequently, there has been a growing interest in studying RNAs without apparent coding function, collectively named noncoding RNAs (ncRNAs). Several ncRNAs, such as ribosomal (r)RNAs, transfer (t)RNAs, small nuclear (sn)RNAs, and small nucleolar (sno)RNAs are well-known for their role in "housekeeping" cell functions (Zhang et al., 2019). In addition, ncRNAs include heterogeneous transcripts with lesser-known regulatory functions, including microRNAs (miRNAs), piwi-interacting (pi)RNAs, small interfering (si)RNAs, and long non-coding (lnc)RNAs (Zhang et al., 2019).

Circular (circ)RNAs are a vast class of ncRNAs with covalently closed ends and lengths between $\sim 100$ to thousands of nucleotides (Kristensen et al., 2019). Unlike lncRNAs, circRNAs are usually well conserved among different species. They mainly originate through a process called backsplicing, in which the 3 ' and 5' ends of a precursor RNA are cleaved and ligated by the splicing machinery. Given the lack of 5' and 3' ends, circRNAs are not degraded by RNA exonucleases and therefore they are quite stable. Most of the circRNAs described to-date originate from exonic sequences (Chen et al., 2015), however they may also contain introns and intergenic sequences. CircRNAs can be localized in both the cytoplasm and the nucleus, as well as outside the cell in extracellular vesicles (Li et al., 2015). They often display a tissue-specific distribution and their expression may be altered in cancer and other pathologies (Kristensen et al., 2019; Liu et al., 2019; Mei et al., 2019; Vo et al., 2019). A growing body of evidence suggests that circRNAs play a role as potential prognostic and diagnostic biomarkers in cancer, given their high stability and specific expression patterns. CircRNAs are present in human body fluids like blood, urine, and saliva, and thus could be easily detected through non-invasive biopsies (Wang et al., 2018; Su et al., 2019; Verduci et al., 2019). 
A number of functions have been described for circRNAs, including synthesis of short polypeptides, recruitment of proteins to DNA, and scaffolding between specific enzymes and substrates (D’Ambra et al., 2019; Kristensen et al., 2019; Lei et al., 2020). Importantly, many circRNAs have been proposed to act as a "sponge" or decoy for microRNAs (miRNAs) and RNA-binding proteins (RBPs) (D’Ambra et al., 2019; Jamal et al., 2019; Kristensen et al., 2019; Verduci et al., 2019). CircRNAs bind and thereby functionally "neutralize" or inactivate miRNAs, thus restoring the translation of proteins that are otherwise suppressed by specific miRNA-mRNA binding events. This process is particularly effective when a circRNA is highly abundant and contains several binding sites for a target miRNA. For example, the well-known circRNA CDR1as (also called ciRS-7) has $>60$ binding sites for miR-7 (Hansen et al., 2013). The sponging of miR-7 by CDR1as has been described in Parkinson's disease, Alzheimer's disease, and several cancers (Shao and Chen, 2016). CircRNAs can also bind RBPs and these interactions may interfere with or enhance the functions of several proteins and mRNAs. For example, circPABPN1 suppressed cell proliferation by interacting with the RBP HuR, preventing HuR from binding to $P A B P N 1$ mRNA, and thereby suppressing the translation of PABPN1, a protein critically involved in cell proliferation (Abdelmohsen et al., 2017). Here, we review the increasingly recognized roles of circRNAs in hematological malignancies (Bonizzato et al., 2016; Mei et al., 2019), with a particular focus on the binding and possible sponging of oncogenic or tumor-suppressive miRNAs. These circRNAs, their effectors, and impacts on hematologic diseases are summarized in Table $\mathbf{1}$ and Figure 1.

\section{circRNAs in AML}

Acute myeloid leukemia (AML) is the most common acute leukemia in adults, with an incidence of over 20,000 cases per year in the United States (De Kouchkovsky and Abdul-Hay, 2016). AML is characterized by the rapid growth of abnormal and immature white blood cells, inhibiting the production of normal hematopoietic cells in the bone marrow.

Many cytogenetic abnormalities causing AML have been characterized and include the large chromosomal translocations $\mathrm{t}(8 ; 21), \mathrm{t}(15 ; 17)$, and $\mathrm{t}(9 ; 11)$, which create the fusion proteins RUNX1-RUNX1T1, PML-RARA, and MLL-AF9, respectively (De Kouchkovsky and Abdul-Hay, 2016). Using patient samples, Guarnerio et al. (2016) found that the rearrangement of chromosomes led to the biogenesis of fusion-circRNAs ( $\mathrm{f}$ circRNAs) and identified two tumor-promoting f-circRNAs, $f$ $\operatorname{circPR}$, and $f$-circ $M 9$, derived from the fusion transcripts $P M L$ RARA and MLL-MLLT3 (AF9), respectively. These f-circRNAs enhanced cell proliferation and promoted leukemogenesis in vivo in mice when co-expressed with their oncogenic fusion protein counterparts. Furthermore, f-circRNAs contributed to therapy resistance by conferring protection from apoptosis during treatment with the chemotherapeutic drugs arsenic trioxide (ATO) and cytarabine (Ara-C).

Cytogenetically normal AML (CN-AML) is not associated with chromosomal aberrations but is characterized by heterogeneous gene mutations with therapeutic and prognostic implications. For instance, mutations in FLT3-IDT (internal tandem duplication in the fms-related tyrosine kinase 3 gene) are associated with a higher risk of relapse, whereas mutations in the chaperone nucleophosmin gene (NPM1) are associated with a favorable prognosis in the absence of other mutations (De Kouchkovsky and Abdul-Hay, 2016). Hirsch et al. (2017) identified several NPM1-derived-circRNAs in $\mathrm{CN}$-AML cell lines carrying either the normal or the mutated NPM1 gene. The circRNA hsa_circ_0075001 was elevated in AML cells independently of the NPM1 mutational status. The levels of hsa_circ_0075001 were higher in a cohort of 46 patients with undifferentiated blasts and correlated negatively with the expression of genes involved in Toll-like receptor (TLR) signaling, which is implicated in hematopoietic cell differentiation (Nagai et al., 2006; Okamoto et al., 2009; Eriksson et al., 2017). Moreover, in patients with high hsa_circ_0075001 levels, the abundance of miR-181 target genes was reduced; the authors linked these two observations by noting that NPM1 mRNA has miR-181 binding sites and circRNAs derived from NPM1 may sequester miR-181 (Hirsch et al., 2017). Importantly, miR-181 is a critical regulator of cellular differentiation and hematological malignancies (Su et al., 2015).

Another circRNA upregulated in CN-AML primary samples, circDLEU2 promoted tumor formation in vivo in mice. High levels of circDLEU2 were proposed to reduce miR-496 function and to promote the expression of PRKACB (Protein Kinase, cAMP-dependent Catalytic $\beta$ ), leading to alterations in cell proliferation and apoptosis (Wu et al., 2018). In CN-AML patients, high levels of another circRNA, circKLHL8, correlated with better overall outcomes, and event-free survival, together with a lower percentage of malignant blasts in blood and bone marrow (Papaioannou et al., 2020). Two other circRNAs, circFOXO3 and circFBXW7, were hypothesized to function as tumor suppressors in AML (Zhou et al., 2019; Papaioannou et al., 2020).

In recent years, additional circRNAs dysregulated in AML have been identified. Chen et al. (2018) found that circANAPC7 (circ_101141) was upregulated in AML bone marrow samples and could play a role in the disease by sponging microRNAs in the miR-181 family, which regulate hematopoietic differentiation. Additional studies were performed on AML bone marrow samples; Ping et al. (2019a) identified circ_0009910 as a circRNA that sponged the tumor-suppressor microRNA miR-20a-5p, thus promoting cancer growth, while Fan et al. (2018) reported that circ_100290 promoted AML cell proliferation and inhibited apoptosis by sponging miR-293, ultimately increasing the expression of Rab10, a member of the oncogenic RAS family. Li et al. (2017) reported the dynamic expression of circ_0004277 in AML patients: circ_0004277 levels were low in newly diagnosed patients compared to healthy controls, but its expression was restored after complete response to induction therapy. Follow-up studies revealed that circ_0004277 levels decreased again during relapse, confirming its potential value as a diagnostic and prognostic biomarker. Bioinformatic analysis predicted that circ_0004277 might be part of a complex network including several miRNAs and mRNAs. 
TABLE 1 | Circular RNAs implicated in hematological malignancies.

\begin{tabular}{|c|c|c|c|c|c|}
\hline & circRNA & Levels & $\begin{array}{l}\text { miRNAs, RBPs, and pathways } \\
\text { targeted }\end{array}$ & Impact on hematologic disease & References \\
\hline \multicolumn{6}{|c|}{ ACUTE MYELOID LEUKEMIA (AML) } \\
\hline$P M L-R A R A$ & $f$-circPR (PML) & de novo & Signaling through AKT & $\begin{array}{l}\text { Increased cell proliferation } \\
\text { Chemotherapy resistance }\end{array}$ & Guarnerio et al., 2016 \\
\hline$M L L-A F 9$ & f-circM9 & de novo & Signaling through MAPK and AKT & $\begin{array}{l}\text { Increased cell proliferation } \\
\text { Chemotherapy resistance }\end{array}$ & Guarnerio et al., 2016 \\
\hline NPM1 & circNPM1 hsa_circ_0075001 & UP & miR-181 TLR signaling & $\begin{array}{l}\text { Altered differentiation } \\
\text { Promotion of leukemogenesis }\end{array}$ & Hirsch et al., 2017 \\
\hline DLEU2 & circDLEU2 hsa_circ_0000488 & UP & miR-496 and PRKACB & $\begin{array}{l}\text { Increased cell proliferation } \\
\text { Inhibition of apoptosis }\end{array}$ & Wu et al., 2018 \\
\hline KLHL8 & circKLHL8 & $\begin{array}{l}\text { Associated } \\
\text { with outcome }\end{array}$ & $\begin{array}{l}\text { miR-155 and increased CDKN1, } \\
\text { CDKN2, BCL6, TLR4, CEBP }\end{array}$ & Positive prognostic marker & Papaioannou et al., 2020 \\
\hline FBXW7 & circFBXW7 & DOWN & $\begin{array}{l}\text { Signal transduction } \\
\text { Leukocyte differentiation }\end{array}$ & Tumor suppression & Papaioannou et al., 2020 \\
\hline FOXO3 & circFOXO3 & DOWN & Apoptotic pathways & $\begin{array}{l}\text { Induced apoptosis } \\
\text { Diagnostic and prognostic biomarker }\end{array}$ & Zhou et al., 2019 \\
\hline ANAPC7 & $\begin{array}{l}\text { circANAPC7 } \\
\text { hsa_circRNA_101141 }\end{array}$ & UP & miR-181 & Possible role in HSCs differentiation & Chen et al., 2018 \\
\hline MFN2 & $\begin{array}{l}\text { circ_0009910 } \\
\text { hsa_circRNA_100053 }\end{array}$ & UP & $\begin{array}{l}\text { miR-20a-5p } \\
\text { Proliferative pathways }\end{array}$ & Cancer growth & Ping et al., $2019 a$ \\
\hline SLC30A7 & circ_100290 & UP & miR-203/Rab10 & $\begin{array}{l}\text { Increased cell proliferation } \\
\text { Inhibition of apoptosis }\end{array}$ & Fan et al., 2018 \\
\hline WDR7 & hsa_cir_0004277 & DOWN & Unconfirmed & Diagnostic and prognostic biomarker & Li et al., 2017 \\
\hline (Unknown) & circ-0004136 & UP & miR-142, miR-29a & $\begin{array}{l}\text { Increased cell proliferation } \\
\text { Inhibition of apoptosis }\end{array}$ & Yuan et al., 2019 \\
\hline VIMENTIN & circVIM & UP & Unknown & Prognostic biomarker & Yi et al., 2019 \\
\hline HIPK2 & circHIPK2 (PML) & DOWN & $\begin{array}{l}\text { miR-124-3p } \\
\text { CEBPA }\end{array}$ & $\begin{array}{l}\text { Prognostic Biomarker } \\
\text { Possible role in differentiation induced by } \\
\text { ATRA treatment }\end{array}$ & Li et al., 2018a \\
\hline (Unknown) & hsa_circ_0004520 & UP & PLXNB2, VEGFA & $\begin{array}{l}\text { Angiogenesis } \\
\text { Prognostic biomarker }\end{array}$ & Lv et al., 2018 \\
\hline MYBL2 & circMYBL2 hsa_circ_0006332 & UP & Enhances FLT3 translation & $\begin{array}{l}\text { Increased cell proliferation and } \\
\text { chemoresistance Inhibition of apoptosis }\end{array}$ & Sun et al., 2019 \\
\hline PAN3 & circPAN3 & UP & $\begin{array}{l}\text { miR-153-5p, miR-183-5p } \\
\text { XIAP }\end{array}$ & $\begin{array}{l}\text { Inhibitor of apoptosis } \\
\text { Increased chemoresistance }\end{array}$ & Shang et al., 2019 \\
\hline \multicolumn{6}{|c|}{ CHRONIC MYELOID LEUKEMIA (CML) } \\
\hline$B C R-A B L 1$ & $f$-circBA9.3 & de novo & Apoptotic pathways & $\begin{array}{l}\text { Negative prognostic factor } \\
\text { Increase chemoresistance }\end{array}$ & Pan et al., 2018 \\
\hline MFN2 & $\begin{array}{l}\text { circ_0009910 } \\
\text { hsa_circRNA_100053 }\end{array}$ & UP & Unconfirmed in CML & $\begin{array}{l}\text { Negative prognostic biomarker } \\
\text { Imatinib resistance }\end{array}$ & Ping et al., 2019b \\
\hline (Unknown) & hsa_circ_0080145 & UP & miR-29b & $\begin{array}{l}\text { Increased cell proliferation } \\
\text { Inhibition of apoptosis }\end{array}$ & Liu et al., 2018 \\
\hline \multicolumn{6}{|c|}{ ACUTE LYMPHOID LEUKEMIA (ALL) } \\
\hline AF4 & circAF4 & UP & $\begin{array}{l}\text { miR-128-3p } \\
\text { MLL-AF4 }\end{array}$ & $\begin{array}{l}\text { Promotes leukemogenesis in vitro and } \\
\text { in vivo }\end{array}$ & Huang et al., 2019 \\
\hline PVT1 & circPVT1 & UP & miR-125, let-7 & $\begin{array}{l}\text { Increased cell proliferation } \\
\text { Inhibition of apoptosis }\end{array}$ & Hu et al., 2018 \\
\hline PAX5 & circPAX5 & UP & miR-124 (unconfirmed) & $\begin{array}{l}\text { May promote } \mathrm{B} \text { cell maturation in pediatric } \\
\text { patients }\end{array}$ & c Gaffo et al., 2019 \\
\hline HIPK3 & circHIPKЗ & UP & miR-124 (unconfirmed) & Unknown & Gaffo et al., 2019 \\
\hline $\begin{array}{l}E N L, A F 6, \\
\text { AF9, AF10, } \\
\text { GAS7 }\end{array}$ & $\begin{array}{l}\text { circENL, circAF6, circAF9, } \\
\text { circAF10, circGAS7 }\end{array}$ & Unknown & Unknown & Unknown & Huang et al., 2019 \\
\hline \multicolumn{6}{|c|}{ CHRONIC LYMPHOID LEUKEMIA (CLL) } \\
\hline$R P L 15$ & circRPL15 hsa_circ_0064574 & UP & $\begin{array}{l}\text { miR-146b-3p } \\
\text { RAF1 }\end{array}$ & $\begin{array}{l}\text { Increased cell proliferation } \\
\text { Diagnostic biomarker }\end{array}$ & Wu et al., 2020 \\
\hline
\end{tabular}


TABLE 1 | Continued

\begin{tabular}{|c|c|c|c|c|c|}
\hline & circRNA & Levels & $\begin{array}{l}\text { miRNA, RBPs, and pathways } \\
\text { targeted }\end{array}$ & Impact on hematologic disease & References \\
\hline$C B F B$ & circCBFB hsa_circ_0000707 & UP & $\begin{array}{l}\text { miR-607 } \\
\text { FZD3, Wnt/ } \beta \text {-catenin } \\
\text { pathway activation }\end{array}$ & $\begin{array}{l}\text { Increased cell proliferation } \\
\text { Inhibition of apoptosis } \\
\text { Prognostic and diagnostic marker }\end{array}$ & Xia et al., 2018 \\
\hline MTO1 & circ_0132266 & DOWN & $\begin{array}{l}\text { miR-337-3p } \\
\text { PML }\end{array}$ & Tumor suppressor & Wu et al., 2019 \\
\hline \multicolumn{6}{|c|}{ LYMPHOMAS } \\
\hline LAMP1 & $\begin{array}{l}\text { circLAMP1 } \\
\text { hsa_circRNA_101303 }\end{array}$ & UP & $\begin{array}{l}\text { miR-615-5p } \\
\text { DDR2 }\end{array}$ & $\begin{array}{l}\text { Increased cell proliferation Inhibition of } \\
\text { apoptosis }\end{array}$ & Deng et al., 2019 \\
\hline$A P C$ & circAPC hsa_circ_0127621 & DOWN & $\begin{array}{l}\mathrm{miR}-888 \\
\text { APC }\end{array}$ & $\begin{array}{l}\text { Tumor suppressor } \\
\text { Diagnostic and prognostic marker }\end{array}$ & Hu et al., 2019 \\
\hline NPM1-ALK & $f$-circNPM1-ALK & de novo & Unknown & Possible diagnostic marker for ALCL & Babin et al., 2018 \\
\hline \multicolumn{6}{|c|}{ MULTIPLE MYELOMA (MM) } \\
\hline Unknown & circ_0000190 & DOWN & $\begin{array}{l}\text { miR-767-5p } \\
\text { MAPK4 }\end{array}$ & $\begin{array}{l}\text { Prognostic marker } \\
\text { Tumor suppressor } \\
\text { Potential therapeutic target }\end{array}$ & Feng et al., 2019 \\
\hline
\end{tabular}

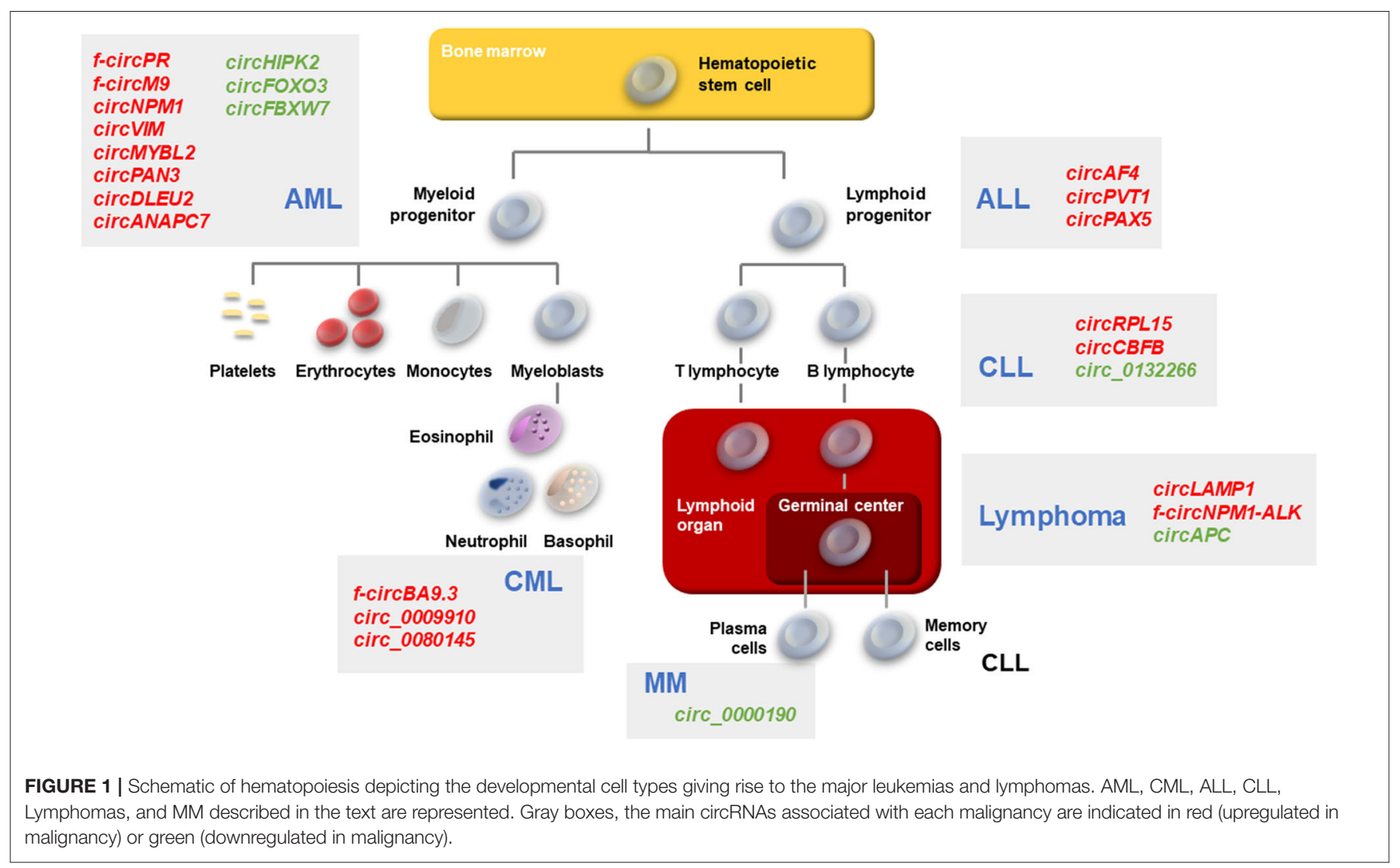

Yuan et al. (2019) reported a rise in circ_0004136 in a pediatric AML cohort and proposed that circ_0004136 promoted cell proliferation at least in part by binding and repressing miR-142, a microRNA known to be associated with pediatric AML. In a cohort of 113 AML patients, Yi et al. (2019) identified circVIM, derived from the VIM (vimentin) gene. Vimentin expression is known to be linked to tumor progression and can be a useful marker of the aggressiveness of certain cancers, such as gastric cancer (Fuyuhiro et al., 2010). Similarly, high levels of circVIM in AML were associated with shorter overall survival and leukemiafree survival, pointing to $\operatorname{circVIM}$ as a possible prognostic marker in AML (Yi et al., 2019).

Acute promyelocytic leukemia (APL), a less common form of AML, is characterized by the formation of the promyelocytic leukemia/retinoic acid receptor $\alpha(\mathrm{PML} / \mathrm{RAR} \alpha)$ fusion protein, which causes many of the features of the disease. APL has a 
high responsiveness to all-trans retinoic acid (ATRA) treatment, which leads to further differentiation and maturation of the leukemic cells (Cicconi et al., 2018; Li et al., 2018a). (Li et al., 2018a) identified several circRNAs differentially expressed in APL-derived NB4 cells during ATRA treatment and validated in patient samples: $\operatorname{circHIPK2}$, $\operatorname{cirCHIPK3}$, $\operatorname{cir} P V T 1$, circRELL1, and circSMARCA5. The authors focused on the levels of circHIPK2, which decline in newly diagnosed persons and are restored after complete remission. Further experiments proved a key relationship between this circRNA and cell maturation: circHIPK2 sponged miR-124-3p and increased the expression of CEBPA, a transcription factor involved in hematopoiesis. Altogether, the results highlighted circHIPK2 as a potential biomarker for APL.

Extramedullary Infiltration (EMI) is a poor prognostic indicator in AML characterized by the accumulation of blasts in extramedullary locations, including spleen, liver, skin, and central nervous system. Lv et al. (2018) found that most circRNAs upregulated in EMI bone marrow samples might be proposed to influence cell adhesion, migration, and signal transduction. Among them, hsa_circ_0004520 is predicted to modulate the expression of VEGFA (vascular endothelial growth factor A), which could contribute to angiogenesis in AML-EMI.

The complexity of molecular and cytogenetic abnormalities is a challenge for the design of AML therapy. While induction therapy with cytarabine and an anthracycline remains a standard of care in AML, resistance may develop over time through different mechanisms (Dombret and Gardin, 2016). Specific tyrosine kinase inhibitors (TKI) have been developed to treat the aforementioned FLT3-ITD AML, but despite success in achieving remission in clinical trials, patients often relapsed or acquired resistance over time (De Kouchkovsky and Abdul-Hay, 2016; Sun et al., 2019). Sun et al. (2019) reported that circMYBL2 silencing restored sensitivity of human FLT3-ITD ${ }^{+}$cells to the TKI quizartinib and inhibited cell proliferation in culture as well as in mice; mechanistically, circMYBL2 enhanced FLT3 translation by facilitating the binding between FLT3 mRNA and the RBP polypyrimidine tract-binding protein 1 (PTBP1).

Shang et al. (2019) identified circPAN3 as a key factor in doxorubicin resistance in AML cell lines (33). CircPAN3 was shown to bind miR-153-3p and miR-183-5p, in turn modulating the expression levels of XIAP (X-linked inhibitor of apoptosis protein), a key protein implicated in autophagy and apoptosis. In addition, circPAN3 downregulation restored drug sensitivity, suggesting a role for this circRNA in AML resistance to conventional chemotherapies.

\section{circRNAs in CML}

Chronic myeloid leukemia (CML) is a rare clonal myeloproliferative malignancy with an annual incidence of one to two cases per 100,000 persons (Zhou and $\mathrm{Xu}, 2015$ ). The cytogenetic hallmark of CML is the Philadelphia $(\mathrm{Ph})$ chromosome, generated by the reciprocal translocation between the long arms of chromosomes 9 and 22, $\mathrm{t}(9 ; 22)$ (q34;q11). The fusion gene $B C R-A B L 1$ arises as a consequence of the translocation and leads to the production of the oncogenic fusion protein BCR-ABL1, a tyrosine kinase that induces the phosphorylation, activation, and dysregulation of signaling molecules involved in the survival and growth of bone marrow progenitor cells (Kang et al., 2016). TKIs are the standard choice of treatment for CML, but their effectiveness depends on the phase of the disease and is challenged by the development of drug resistance over time (Litwinska and Machalinski, 2017; Patel et al., 2017). High levels of BCR-ABL1 kinase activity may arise as a consequence of gene amplification and $\mathrm{Ph}$ duplication, and may be sufficient to confer TKI resistance. In addition, specific mutations in the kinase domain (KD) of BCR-ABL1 may enhance drug resistance (Soverini et al., 2014; Patel et al., 2017).

Pan et al. (2018) identified f-circRNA circBA9.3, derived from the $B C R-A B L 1 \mathrm{mRNA}$, as potentially involved in drug resistance to Imatinib, a second-generation TKI. CircBA9.3 expression was higher in TKI-resistant patients compared to responsive controls. Moreover, $\operatorname{circBA9} 9.3$ transfection in BCR-ABL-negative cell lines enhanced proliferation and cancer progression. Further experiments confirmed that circBA9.3 levels were positively correlated with the expression of BCR-ABL1. Regardless of whether first or second generation TKIs were administered, cells overexpressing circBA9.3 displayed less apoptosis compared to controls.

Further studies investigated the role of circRNAs in CML drug resistance. Ping et al. (2019b) used circRNA microarrays to assess circRNA profiles in CML and found that hsa_circ_100053 levels increased in both cells and serum of CML patients. In keeping with the proposal that $h s a_{-}$circ_100053 might be a potential biomarker in CML, higher expression of hsa_circ_100053 was associated with advanced clinical stage, BCR/ABL1 mutational status and resistance to Imatinib. High levels of hsa_circ_100053 were suggested as a negative prognostic factor in the overall survival of CML patients.

Through an RNA-sequencing screen, Liu et al. (2018) identified hsa_circ_0080145 as being upregulated in cells from CML patients and in cell lines K562 and KU812. Moreover, hsa_circ_0080145 silencing suppressed leukemic cell proliferation. In functional experiments, the authors found that hsa_circ_0080145 was capable of sponging miR$29 \mathrm{~b}$, and the targets of miR-29b were predicted with the tool miRTarBase (http://miRTarBase.mbc.nctu.edu.tw/), which contains interactions validated experimentally. Gene ontology analysis revealed that these genes belong to three main groups: systemic lupus erythematosus pathway, cAMP signaling pathway, and heterocycle biosynthetic process. In agreement with these findings, a previous study reported that miR-29b was downregulated in CML and overexpression of miR-29b in K562 cells inhibited leukemic cell growth and promoted apoptosis through regulation of the BCR-ABL1 tyrosine kinase (Li et al., 2013). In addition, several other oncogenes could be potentially silenced by miR-29, including the antiapoptotic protein MCL1, upstream inhibitors of p53, DNA methyltransferases, and extracellular matrix proteins (Li et al., 2013).

Together, these findings highlight the importance of circRNAs as potential CML biomarkers with key roles in drug resistance and as targets for new therapeutic treatments. 


\section{circRNAs in ALL}

Acute lymphoblastic leukemia (ALL) is the most common cancer among children in the USA and the most frequent cause of cancer death in young people (Hunger and Mullighan, 2015). ALL may arise from B-cell (B-ALL) or T-cell (TALL) precursors. Current medical treatments for ALL allow high survival rates, but relapse occurs in $15-20 \%$ of pediatric patients and is associated with a higher risk of treatment failure (Schrappe et al., 2012). Most somatic mutations acquired in ALL are chromosome rearrangements, such as translocations and hyperdiploidy. Translocation $\mathrm{t}(4 ; 11)(\mathrm{q} 21 ; \mathrm{q} 23)$ resulting in the chimeric product MLL-AF4 is commonly identified in infant pro-B-ALL and has poor prognosis (Mrózek et al., 2009).

The first studies on circRNAs differentially expressed in ALL (Salzman et al., 2012) found that $>10 \%$ of the transcripts encoded by hundreds of genes in naive B cells (CD19+) and hematopoietic stem cells (CD34+) were circRNAs. Subsequently, Huang et al. (2019) identified different circRNAs derived from the MLL partner fusion gene AF4. The levels of circAF4(ex34) were higher in the leukemia cell line analyzed (RS4;11) and patients under 8 years of age. Moreover, $\operatorname{circAF} 4$ levels correlated with the severity of disease, and circAF4 silencing led to increased apoptosis in cells carrying the $M L L-A F 4$ translocation. In mice, circAF4 knockdown improved survival and reduced spleen infiltration. By binding miR-128-3p, circAF4 might sequester the microRNA away from the fusion $M L L-A F 4$ mRNA and enable MLL-AF4 expression. Simultaneous circAF4 silencing and miR128-3p overexpression in vivo supported this regulatory axis and suggested that circAF4 acts as an oncogenic circRNA in leukemia. In a further study, Dal Molin et al. (2019) found that specific rearrangements leading to fusions between $M L L$ and other genes not only generated alternative isoforms of circRNAs in different subtypes of leukemias, but may also contribute to the production of disease-associated aberrant circRNAs.

Hu et al. (2018) found that circPVT1 dysregulation promoted cell proliferation and inhibited apoptosis in ALL cell lines by sponging miR-125 and let-7, ultimately increasing the expression of the oncogene MYC and the anti-apoptotic protein BCL2. Other studies showed that $\operatorname{circPVT1}$ regulated the function of let7 family members which function as tumor suppressors (Panda et al., 2017a). CircPVT1 had been previously described as a negative prognostic factor for gastric cancer (Chen et al., 2017a). In sum, by interfering with let-7 function, $\operatorname{circPVT1}$ was proposed to promote ALL leukemogenesis (Hu et al., 2018).

Gaffo et al. (2019) identified and quantified bioinformatically the circRNAs expressed in $\mathrm{T}$ cells, $\mathrm{B}$ cells and monocytes in physiological conditions; as expected, circRNA signatures varied with stage of differentiation and cell type. Further analysis of circRNAs differentially expressed in B-cell precursors of ALL pediatric patients found upregulated circPVT1, circHIPK3, and circPAX5. The PAX5 (paired box protein five) gene encodes transcription factor BSAP (Bcell-lineage-specific activator protein), with a key role in defining and maintaining B-cell identity. The binding of circPAX5 and circHIPK3 to miR-124-5p was proposed to synergistically interfere with $B$ cell maturation and promote disease progression.

\section{circRNAs in CLL}

Chronic lymphocytic leukemia (CLL) is characterized by the accumulation of small and mature $\mathrm{CD}^{+} \mathrm{B}$ cells in blood, bone marrow and secondary lymphoid organs. CLL B-cells have a relatively low proliferation rate and high resistance to apoptosis (Kipps et al., 2017). The molecular classification of the disease relies on whether the neoplastic cells express a mutated or unmutated form of the immunoglobulin heavy chain variable region gene $(I G H V)$, with the unmutated IGHV being a marker of poor prognosis and shorter survival. The variability in clinical behaviors reflects the underlying genetic heterogeneity (Kipps et al., 2017). To date, three main circRNAs have been studied in CLL: circRPL15 (Wu et al., 2020), circCBFB (Xia et al., 2018), and circ_0132266 (Wu et al., 2019).

CircRPL15 was evaluated as a potential biomarker for the diagnostic screening in plasma from patients with CLL, especially in cases without IGHV mutation (Wu et al., 2020). Upregulated circRPL15 was proposed to sponge miR-146b-3p, thereby increasing RAF1 protein levels. As an effector of the proliferative RAS pathway, RAF1 could in turn phosphorylate and thereby activate MAPK (mitogen-activated protein kinase) signaling, promoting cell growth. In support of this paradigm, knockdown of circRPL15 in human cell lines reduced the phosphorylation of mitogenic factors (Wu et al., 2020) and an earlier study by Wang et al. (2008) found that RAF1 was overexpressed in CLL.

After finding that $\operatorname{circ} C B F B$, derived from $C B F B$ (core-binding factor subunit beta) pre-mRNA, was upregulated in untreated CLL cells from patients, Xia et al. (2018) proposed that it could serve as a prognostic and diagnostic marker in CLL patients. Mechanistically, $\operatorname{circCBFB}$ was found to activate the Wnt/ßcatenin pathway in human cell lines by binding miR-607 and thereby derepressing production of Frizzled (FZD3), a receptor for Wnt; the ensuing proliferative and anti-apoptotic phenotype could therefore be involved in CLL progression. Moreover, higher levels of $\operatorname{circCBFB}$ and $\operatorname{circRPL} 15$ correlated with poor overall survival and shorter survival time.

$\mathrm{Wu}$ et al. (2019) proposed a tumor-suppressor role for circ_0132266 in CLL based on the ability of circ_0132266 to bind miR-337-3p in CLL cell lines. PML (promyelocytic leukemia protein), a broad regulator of gene expression and cell viability, is a key target of miR-337-3p. The authors linked the reduction in circ_0132266 levels to the increased miR-337-3p levels and proposed that by sponging miR-337-3p, circ_0132266 might be tumor-suppressive in CLL.

\section{circRNAs in Lymphomas}

Lymphomas originate from lymphocytes or their progenitor cells and tend to be localized in lymph nodes and the lymphatic system. Due to the high heterogeneity of lymphocytes, given the diverse lineages from which they arise, the stages of differentiation, and their specific functions, the classification of lymphomas is quite complex. Lymphomas are classified into Hodgkin and non-Hodgkin, the latter being more common; the non-Hodgkin's lymphoma can be divided into two major groups depending on the cell lineage: B-cell and T-cell lymphomas (Swerdlow et al., 2016). 
In T-cell lymphoblastic lymphoma (T-LBL) cell lines, Deng et al. (2019) described a regulatory axis in which circLAMP1 was proposed to sponge miR-615-5p, thereby increasing the levels of DDR2 (discoidin domain receptor tyrosine kinase 2). DDR2 encodes a member of the receptor tyrosine kinase (RTKs) protein family, is induced by collagen, and in turn activates signal transduction pathways involved in proliferation, extracellular matrix remodeling, wound repair, and tumor invasiveness. Gainand loss-of function experiments supported a role for circLAMP1 in promoting cell proliferation and repressing apoptosis in $\mathrm{T}$ LBL cells.

Diffuse large B-cell lymphoma (DLBCL) is the most common type of non-Hodgkin lymphoma worldwide (Li et al., 2018b). Morphologically, it is characterized by large B cells arranged in a diffuse pattern. RNA-seq analysis of DLBCL identified several genetic abnormalities and different mutation profiles. At present, the standard of care received by DLBCL patients is based on a combination of a monoclonal antibody (rituximab) and chemotherapy, a regimen called R-CHOP. The current 5-year overall survival rate is estimated around 60\% (Li and Medeiros, 2018). CircAPC, downregulated in DLBCL cell lines and plasma from patients (Hu et al., 2019), originates from the backsplicing of the linear adenomatous polyposis coli $(A P C)$ gene, encoding a major regulator of cell proliferation. The tumor suppressor functions of the protein APC include regulation of cell adhesion, inhibition of Wnt/ $\beta$ catenin pathway and control of cell cycle progression (Zhang and Shay, 2017). Importantly, DLBCL usually affects the gastrointestinal tract, where APC expression is most prominent (Li et al., 2018b). CircAPC was detected in both the cytoplasm and the nucleus of DLBCL cells (Hu et al., 2019). In the nucleus, circAPC bound the APC promoter and recruited DNA demethylase Ten-Eleven Translocation 1 (TET1), thereby reducing methylation and increasing $A P C$ transcription. In the cytoplasm, circAPC sequestered miR-888, enhancing APC translation and eventually inhibiting the proliferative Wnt/ $\beta$ catenin pathway. In short, reduced levels of circAPC were proposed to promote DLBCL progression. Further analysis pointed to circAPC as a diagnostic and prognostic tool; for instance, low circAPC levels correlated with resistance to R-CHOP treatment, low International Prognostic Index (IPI) and advanced staging (formulated with the Ann-Harbor staging classification). Ectopic circAPC expression was shown to suppress DLBCL proliferation in vitro and in vivo in a mouse xenograft model.

ALCL (Anaplastic Large Cell Lymphoma) is a rare but highly aggressive T-cell non-Hodgkin lymphoma that originates from a chromosomal translocation that produces the fusion protein NPM1-ALK, a constitutively active tyrosine kinase (Fuchs et al., 2019). Babin et al. (2018) employed the CRISPR/Cas9 technology to generate the same translocation in different mouse and human cell lines and identified novel $f$-circRNAs, specifically f-circRNA-mA and $f$-circRNA-hD (collectively called f-circRNA-NPM1-ALK). This pool of circRNAs was also identified in some of the original tumors, thus representing a possible diagnostic marker for ALCL.

\section{circRNAs in MM}

Multiple myeloma (MM) is characterized by proliferation of immunoglobulin-secreting plasma cells within the bone marrow. It is the second most common hematological cancer, accounting for $10 \%$ of all blood malignancies (Walker et al., 2014), with a life expectancy ranging from a few months to $>10$ years and hampered by an extremely rate of metastasis and drug resistance (Palumbo et al., 2015). High-risk cytogenetic subgroups, including those with deletion of chromosome 17p or gain of chromosome 1q21, progress rapidly and are associated with shortened overall survival (Sherbenou et al., 2016).

Feng et al. (2019) found that circ_0000190 was downregulated in bone marrow biopsies and peripheral blood derived from MM patients. The authors propose that the reduction in circ_0000190 led to increased levels and function of miR767-5p, which in turn lowered the levels of MAPK4, an inhibitor of MM progression. Analysis of 47 patients revealed that higher expression of circ_0000190 was associated with longer progression-free disease and improved overall survival. In murine xenograft models, ectopic expression of circ_0000190 reduced tumor progression by $60 \%$, underscoring the potential therapeutic value of this circRNA. Interestingly, the tumorsuppressor role of circ_0000190 was originally described in gastric cancer (Chen et al., 2017b).

\section{CLOSING REMARKS AND CHALLENGES AHEAD}

In this review, we have highlighted the progress made in identifying circRNAs implicated in hematological malignancies. Although many functions have been proposed for circRNAs (Panda et al., 2017b), most of the examples to-date underscore their ability to bind other molecules and sequester them away from miRNAs and RBPs. Through these specific interactions, circRNAs may influence the levels of proteins under the control of such miRNAs and RBPs. As uncovered in this review, many of these target proteins are implicated in controlling cell proliferation and survival. In the context of hematological diseases, dysregulation of RBPs and miRNAs has been linked to the initiation and progression of malignancy, chemotherapy resistance, and poor clinical outcome. To apply the emerging knowledge of circRNAs as potential interventions in the progression and treatment of leukemias and lymphomas, we have identified several challenges.

First, superior methods must be developed to identify and quantify circRNAs in all systems, including hematologic malignancies. More complete databases with comprehensive circRNA annotations are needed, particularly those that include information on tissue-specific circRNAs. In this regard, normalization of circRNA nomenclature will be particularly helpful to improve clarity across studies. Molecular methodologies must also improve to offer more sensitive and specific means of detecting circRNAs with diagnostic and prognostic value, given that they are often in quite low abundance. 
Second, the field must adopt more rigorous methods of elucidating circRNA function and circRNA-interacting molecules. While the notion of circRNAs as "microRNA sponges" is certainly attractive, few studies have included the careful stoichiometric measurements and molecular biology interventions to demonstrate that a circRNA is indeed a sponge for an interacting microRNA. These careful analyses are particularly important, since many circRNAs exist in low copy numbers (often one or less per cell) while microRNAs often exist in hundreds or more copies per cell, so the sponging model may not be correct in every case.

Third, hematopoietic malignancies are particularly well suited for RNA-directed therapies such as antisense oligomers (ASOs), given their immediate access in the blood. However, the fact that the vast majority of circRNAs share most of their sequence with the parent linear RNA challenges these efforts. Improved bioinformatic and molecular approaches to optimize the targeting of the junction sequences of circRNAs will enable more accurate targeting of circRNAs for elimination in therapeutic settings. Conversely, for circRNAs with beneficial effects in hematopoietic malignancies, ectopic delivery methods could have therapeutic advantage; such delivery methods

\section{REFERENCES}

Abdelmohsen, K., Panda, A. C., Munk, R., Grammatikakis, I., Dudekula, D. B., De, S., et al. (2017). Identification of HuR target circular RNAs uncovers suppression of PABPN1 translation by CircPABPN1. RNA Biol. 14, 361-369. doi: 10.1080/15476286.2017.1279788

Babin, L., Piganeau, M., Renouf, B., Lamribet, K., Thirant, C., Deriano, L., et al. (2018). Chromosomal translocation formation is sufficient to produce fusion circular RNAs specific to patient tumor cells. iScience 5, 19-29. doi: 10.1016/j.isci.2018.06.007

Bonizzato, A., Gaffo, E., Te Kronnie, G., and Bortoluzzi, S. (2016). CircRNAs in hematopoiesis and hematological malignancies. Blood Cancer J. 6:e483. doi: $10.1038 /$ bcj.2016.81

Chen, H., Liu, T., Liu, J., Feng, Y., Wang, B., Wang, J., et al. (2018). Circ-ANAPC7 is upregulated in acute myeloid leukemia and appears to target the MiR-181 family. Cell. Physiol. Biochem. 47, 1998-2007. doi: 10.1159/000491468

Chen, I., Chen, C. Y., and Chuang, T. J. (2015). Biogenesis, identification, and function of exonic circular RNAs. Wiley Interdiscip Rev RNA 6, 563-579. doi: 10.1002/wrna.1294

Chen, J., Li, Y., Zheng, Q., Bao, C., He, J., Chen, B., et al. (2017a). Circular RNA profile identifies circPVT1 as a proliferative factor and prognostic marker in gastric cancer. Cancer Lett. 388, 208-219. doi: 10.1016/j.canlet.2016.12.006

Chen, S., Li, T., Zhao, Q., Xiao, B., and Guo, J. (2017b). Using circular RNA hsa_circ_0000190 as a new biomarker in the diagnosis of gastric cancer. Clin. Chim. Acta 466, 167-171. doi: 10.1016/j.cca.2017.01.025

Cicconi, L., Fenaux, P., Kantarjian, H., Tallman, M., Sanz, M. A., and Lo-Coco, F. (2018). Molecular remission as a therapeutic objective in acute promyelocytic leukemia. Leukemia 32, 1671-1678. doi: 10.1038/s41375-018-0219-5

Dal Molin, A., Bresolin, S., Gaffo, E., Tretti, C., Boldrin, E., Meyer, L. H., et al. (2019). CircRNAs are here to stay: a perspective on the MLL recombinome. Front. Genet.10:88. doi: 10.3389/fgene.2019.00088

D'Ambra, E., Capauto, D., and Morlando, M. (2019). Exploring the regulatory role of circular RNAs in neurodegenerative disorders. Int. J. Mol. Sci. 20:5477. doi: 10.3390/ijms20215477

De Kouchkovsky, I., and Abdul-Hay, M. (2016). Acute myeloid leukemia: a comprehensive review and 2016 update. Blood Cancer J. 6:e441. doi: $10.1038 /$ bcj. 2016.50 also await extensive development and optimization for use in the clinic.

In closing, we are gaining tools and knowledge of circRNAs implicated in hematologic cancers. The expansion and refinement of these tools and knowledge in the near future will enable the development of effective means of diagnosis, prognosis, and therapy directed at circRNAs in leukemias, lymphomas, and other hematologic malignancies.

\section{AUTHOR CONTRIBUTIONS}

\author{
OP, MR, and MG wrote the paper.
}

\section{FUNDING}

This work was supported entirely by the National Institute on Aging Intramural Research Program, National Institutes of Health.

\section{ACKNOWLEDGMENTS}

We thank R. Munk and J. L. Martindale for critical reading of the manuscript.

Deng, L., Liu, G., Zheng, C., Zhang, L., Kang, Y., and Yang, F. (2019). CircLAMP1 promotes T-cell lymphoblastic lymphoma progression via acting as a ceRNA for miR-615-5p to regulate DDR2 expression. Gene 701, 146-151. doi: 10.1016/j.gene.2019.03.052

Dombret, H., and Gardin, C. (2016). An update of current treatments for adult acute myeloid leukemia. Blood 127, 53-61. doi: 10.1182/blood-2015-08-604520

ENCODE Project Consortium (2012). An integrated encyclopedia of DNA elements in the human genome. Nature 489, 57-74. doi: 10.1038/nature11247

Eriksson, M., Peña-Martínez, P., Ramakrishnan, R., Chapellier, M., Högberg, C., Glowacki, G., et al. (2017). Agonistic targeting of TLR1/TLR2 induces p38 MAPK-dependent apoptosis and NFKB-dependent differentiation of AML cells. Blood Adv. 1, 2046-2057. doi: 10.1182/bloodadvances.2017006148

Fan, H., Li, Y., Liu, C., Liu, Y., Bai, J., and Li, W. (2018). Circular RNA-100290 promotes cell proliferation and inhibits apoptosis in acute myeloid leukemia cells via sponging miR-203. Biochem. Biophys. Res. Commun. 507, 178-184. doi: 10.1016/j.bbrc.2018.11.002

Feng, Y., Zhang, L., Wu, J., Khadka, B., Fang, Z., Gu, J., et al. (2019). CircRNA circ_0000190 inhibits the progression of multiple myeloma through modulating miR-767-5p/MAPK4 pathway. J. Exp. Clin. Cancer Res. 38:54. doi: 10.1186/s13046-019-1071-9

Fuchs, S., Naderi, J., and Meggetto, F. (2019). Non-coding RNA networks in ALK-positive anaplastic-large cell lymphoma. Int. J. Mol. 20:2150. doi: 10.3390/ijms20092150

Fuyuhiro, Y., Yashiro, M., Noda, S., Kashiwagi, S., Matsuoka, J., Doi, Y., et al. (2010). Clinical significance of vimentin-positive gastric cancer cells. Anticancer Res. 30, 5239-5243.

Gaffo, E., Boldrin, E., Dal Molin, A., Bresolin, S., Bonizzato, A., Trentin, L., et al. (2019). Circular RNA differential expression in blood cell populations and exploration of circRNA deregulation in pediatric acute lymphoblastic leukemia. Sci. Rep. 9:14670. doi: 10.1038/s41598-019-50864-z

Guarnerio, J., Bezzi, M., Jeong, J. C., Paffenholz, S. V., Berry, K., Naldini, M. M., et al. (2016). Oncogenic role of fusion-circRNAs derived from cancer-associated chromosomal translocations. Cell 165, 289-302. doi: 10.1016/j.cell.2016.03.020

Hansen, T. B., Jensen, T. I., Clausen, B. H., Bramsen, J. B., Finsen, B., Damgaard, C. K., et al. (2013). Natural RNA circles function as efficient microRNA sponges. Nature 495, 384-388. doi: 10.1038/nature11993 
Hirsch, S., Blätte, T. J., Grasedieck, S., Cocciardi, S., Rouhi, A., JongenLavrencic, M., et al. (2017). Circular RNAs of the nucleophosmin (NPM1) gene in acute myeloid leukemia. Haematologica 102, 2039-2047. doi: 10.3324/haematol.2017.172866

Hu, J., Han, Q., Gu, Y., Ma, J., McGrath, M., Qiao, F., et al. (2018). Circular RNA PVT1 expression and its roles in acute lymphoblastic leukemia. Epigenomics 10, 723-732. doi: 10.2217/epi-2017-0142

Hu, Y., Zhao, Y., Shi, C., Ren, P., Wei, B., Guo, Y., et al. (2019). A circular RNA from APC inhibits the proliferation of diffuse large B-cell lymphoma by inactivating Wnt/ $\beta$-catenin signaling via interacting with TET1 and miR-888. Aging 11, 8068-8084. doi: 10.18632/aging.102122

Huang, W., Fang, K., Chen, T. Q., Zeng, Z. C., Sun, Y. M., Han, C., et al. (2019). circRNA circAF4 functions as an oncogene to regulate MLL-AF4 fusion protein expression and inhibit MLL leukemia progression. J. Hematol. Oncol. 12:103. doi: 10.1186/s13045-019-0800-z

Hunger, S. P., and Mullighan, C. G. (2015). Acute lymphoblastic leukemia in children. N. Engl. J. Med. 373, 1541-1552. doi: 10.1056/NEJMra1400972

Jamal, M., Song, T., Chen, B., Faisal, M., Hong, Z., Xie, T., et al. (2019). Recent progress on circular RNA research in acute myeloid leukemia. Front. Oncol. 9:1108. doi: 10.3389/fonc.2019.01108

Kang, Z. J., Liu, Y. F., Xu, L. Z., Long, Z. J., Huang, D., Yang, Y., et al. (2016). The philadelphia chromosome in leukemogenesis. Chin. J. Cancer 35:48. doi: 10.1186/s40880-016-0108-0

Kipps, T. J., Stevenson, F. K., Wu, C. J., Croce, C. M., Packham, G., Wierda, W. G., et al. (2017). Chronic lymphocytic leukaemia. Nat. Rev. Dis. Primers 3:96. doi: 10.1038/nrdp.2016.96

Kristensen, L. S., Andersen, M. S., Stagsted, L., Ebbesen, K. K., Hansen, T. B., and Kjems, J. (2019). The biogenesis, biology and characterization of circular RNAs. Nat. Rev. Genet. 20, 675-691. doi: 10.1038/s41576-019-0158-7

Lei, M., Zheng, G., Ning, Q., Zheng, J., and Dong, D. (2020). Translation and functional roles of circular RNAs in human cancer. Mol. Cancer 19:30. doi: 10.1186/s12943-020-1135-7

Li, S., Ma, Y., Tan, Y., Ma, X., Zhao, M., Chen, B., et al. (2018a). Profiling and functional analysis of circular RNAs in acute promyelocytic leukemia and their dynamic regulation during all-trans retinoic acid treatment. Cell Death Dis. 9:651. doi: 10.1038/s41419-018-0699-2

Li, S., Young, K. H., and Medeiros, L. J. (2018b). Diffuse large B-cell lymphoma. Pathology 50, 74-87. doi: 10.1016/j.pathol.2017.09.006

Li, W., Zhong, C., Jiao, J., Li, P., Cui, B., Ji, C., et al. (2017). Characterization of hsa_circ_0004277 as a new biomarker for acute myeloid leukemia via circular RNA profile and bioinformatics analysis Int. J. Mol. 18:597. doi: 10.3390/ijms 18030597

Li, Y., Wang, H., Tao, K., Xiao, Q., Huang, Z., Zhong, L., et al. (2013). miR-29b suppresses CML cell proliferation and induces apoptosis via regulation of BCR/ABL1 protein. Exp. Cell Res. 319, 1094-1101. doi: 10.1016/j.yexcr.2013.02.002

Li, Y., Zheng, Q., Bao, C., Li, S., Guo, W., Zhao, J., et al. (2015). Circular RNA is enriched and stable in exosomes: a promising biomarker for cancer diagnosis. Cell Res. 25, 981-984. doi: 10.1038/cr.2015.82

Litwinska, Z., and Machalinski, B. (2017). miRNAs in chronic myeloid leukemia: small molecules, essential function. Leuk. Lymphoma 58, 1297-1305. doi: 10.1080/10428194.2016.1243676

Liu, J., Kong, F., Lou, S., Yang, D., and Gu, L. (2018). Global identification of circular RNAs in chronic myeloid leukemia reveals hsa_circ_0080145 regulates cell proliferation by sponging miR-29b. Biochem. Biophys. Res. Commun. 504, 660-665. doi: 10.1016/j.bbrc.2018.08.154

Liu, Y., Cheng, Z., Pang, Y., Cui, L., Qian, T., Quan, L., et al. (2019). Role of microRNAs, circRNAs and long noncoding RNAs in acute myeloid leukemia. J. Hematol. Oncol. 12:51. doi: 10.1186/s13045-019-0 734-5

Lv, C., Sun, L., Guo, Z., Li, H., Kong, D., Xu, B., et al. (2018). Circular RNA regulatory network reveals cell-cell crosstalk in acute myeloid leukemia extramedullary infiltration. J. Transl. Med. 16:361. doi: 10.1186/s12967-018-1726-x

Mei, M., Wang, Y., Li, Z., and Zhang, M. (2019). Role of circular RNA in hematological malignancies. Oncol. Lett. 18, 4385-4392. doi: $10.3892 / 0 l .2019 .10836$
Mrózek, K., Radmacher, M. D., Bloomfield, C. D., and Marcucci, G. (2009) Molecular signatures in acute myeloid leukemia. Curr. Opin. Hematol. 16, 64-69. doi: 10.1097/MOH.0b013e3283257b42

Nagai, Y., Garrett, K. P., Ohta, S., Bahrun, U., Kouro, T., Akira, S., et al. (2006). Toll-like receptors on hematopoietic progenitor cells stimulate innate immune system replenishment. Immunity 24, 801-812. doi: 10.1016/j.immuni.2006.04.008

Okamoto, M., Hirai, H., Taniguchi, K., Shimura, K., Inaba, T., Shimazaki, C., et al. (2009). Toll-like receptors (TLRs) are expressed by myeloid leukaemia cell lines, but fail to trigger differentiation in response to the respective TLR ligands. $B r$. J. Hematol. 147, 585-587. doi: 10.1111/j.1365-2141.2009.07858.x

Palumbo, A., Avet-Loiseau, H., Oliva, S., Lokhorst, H. M., Goldschmidt, H., Rosinol, L., et al. (2015). Revised international staging system for multiple myeloma: a report from international myeloma working group. J. Clin. Oncol. 33, 2863-2869. doi: 10.1200/JCO.2015.61.2267

Pan, Y., Lou, J., Wang, H., An, N., Chen, H., Zhang, Q., et al. (2018). CircBA9.3 supports the survival of leukaemic cells by up-regulating c-ABL1 or BCR-ABL1 protein levels. Blood Cells Mol. Dis. 73, 38-44. doi: 10.1016/j.bcmd.2018.09.002

Panda, A. C., Grammatikakis, I., Kim, K. M., De, S., Martindale, J. L., Munk, R., et al. (2017a). Identification of senescence-associated circular RNAs (SAC-RNAs) reveals senescence suppressor CircPVT1. Nucleic Acids Res. 45, 4021-4035. doi: 10.1093/nar/gkw1201

Panda, A. C., Grammatikakis, I., Munk, R., Gorospe, M., and Abdelmohsen, K. (2017b). Emerging roles and context of circular RNAs. Wiley Interdiscip Rev RNA. 8:1386. doi: 10.1002/wrna.1386

Papaioannou, D., Volinia, S., Nicolet, D., Swierniak, M., Petri, A., Mrózek, K., et al. (2020). Clinical and functional significance of circular RNAs in cytogenetically normal AML. Blood Adv. 4, 239-251. doi: 10.1182/bloodadvances.2019000568

Patel, A. B., O'Hare, T., and Deininger, M. W. (2017). Mechanisms of resistance to ABL kinase inhibition in chronic myeloid leukemia and the development of next generation ABL kinase inhibitors. Hematol. Oncol. Clin. North Am. 31 , 589-612. doi: 10.1016/j.hoc.2017.04.007

Ping, L., Jian-Jun, C., Chu-Shu, L., Guang-Hua, L., and Ming, Z. (2019a). Silencing of circ_0009910 inhibits acute myeloid leukemia cell growth through increasing miR-20a-5p. Blood Cells Mol. Dis. 75, 41-47. doi: 10.1016/j.bcmd.2018.12.006

Ping, L., Jian-Jun, C., Chu-Shu, L., Guang-Hua, L., and Ming, Z. (2019b). High circ_100053 predicts a poor outcome for chronic myeloid leukemia and is involved in imatinib resistance. Oncol. Res. doi: 10.3727/096504018X15412701483326. [Epub ahead of print].

Salzman, J., Gawad, C., Wang, P. L., Lacayo, N., and Brown, P. O. (2012). Circular RNAs are the predominant transcript isoform from hundreds of human genes in diverse cell types. PLoS ONE 7:e30733. doi: 10.1371/journal.pone.0030733

Schrappe, M., Hunger, S. P., Pui, C. H., Saha, V., Gaynon, P. S., Baruchel, A., et al. (2012). Outcomes after induction failure in childhood acute lymphoblastic leukemia. N. Engl. J. Med. 366, 1371-1381. doi: 10.1056/NEJMoa1110169

Shang, J., Chen, W. M., Liu, S., Wang, Z. H., Wei, T. N., Chen, Z. Z., et al. (2019). CircPAN3 contributes to drug resistance in acute myeloid leukemia through regulation of autophagy. Leuk. Res. 85:106198. doi: 10.1016/j.leukres.2019.106198

Shao, Y., and Chen, Y. (2016). Roles of circular RNAs in neurologic disease. Front. Mol. Neurosci. 9:25. doi: 10.3389/fnmol.2016.00025

Sherbenou, D. W., Aftab, B. T., Su, Y., Behrens, C. R., Wiita, A., Logan, A. C., et al. (2016). Antibody-drug conjugate targeting CD46 eliminates multiple myeloma cells. J. Clin. Invest. 126, 4640-4653. doi: 10.1172/JCI85856

Soverini, S., Branford, S., Nicolini, F. E., Talpaz, M., Deininger, M. W., Martinelli, G., et al. (2014). Implications of BCR-ABL1 kinase domainmediated resistance in chronic myeloid leukemia. Leuk. Res. 38, 10-20. doi: 10.1016/j.leukres.2013.09.011

Su, M., Xiao, Y., Ma, J., Tang, Y., Tian, B., Zhang, Y., et al. (2019). Circular RNAs in Cancer: emerging functions in hallmarks, stemness, resistance and roles as potential biomarkers. Mol. Cancer. 18:90. doi: 10.1186/s12943-019-1002-6

Su, R., Lin, H. S., Zhang, X. H., Yin, X. L., Ning, H. M., Liu, B., et al. (2015). MiR-181 family: regulators of myeloid differentiation and acute myeloid leukemia as well as potential therapeutic targets. Oncogene 34, 3226-3239. doi: 10.1038/onc.2014.274

Sun, Y. M., Wang, W. T., Zeng, Z. C., Chen, T. Q., Han, C., Pan, Q., et al. (2019). circMYBL2, a circRNA from MYBL2, regulates FLT3 translation 
by recruiting PTBP1 to promote FLT3-ITD AML progression. Blood 134, 1533-1546. doi: 10.1182/blood.2019000802

Swerdlow, S. H., Campo, E., Pileri, S. A., Harris, N. L., Stein, H., Siebert, R., et al. (2016). The 2016 revision of the world health organization classification of lymphoid neoplasms. Blood 127, 2375-2390. doi: 10.1182/blood-2016-01-643569

Verduci, L., Strano, S., Yarden, Y., and Blandino, G. (2019). The circRNAmicroRNA code: emerging implications for cancer diagnosis and treatment. Mol. Oncol. 13, 669-680. doi: 10.1002/1878-0261.12468

Vo, J. N., Cieslik, M., Zhang, Y., Shukla, S., Xiao, L., Zhang, Y., et al. (2019). The landscape of circular RNA in cancer. Cell 176, 869-881.e13. doi: 10.1016/j.cell.2018.12.021

Walker, R. E., Lawson, M. A., Buckle, C. H., Snowden, J. A., and Chantry, A. D. (2014). Myeloma bone disease: pathogenesis, current treatments and future targets. Br. Med. Bull. 111, 117-138. doi: 10.1093/bmb/ldu016

Wang, M., Kluin, P., Rosati, S., Luinge, M., Daenen, S., and Visser, L. (2008). Raf-1 is overexpressed in chronic lymphocytic leukemia and promotes cell survival by phosphorylation of ERK and Bad. (dissertation/thesis). University of Groningen, Groningen, Netherlands. doi: 10.1182/blood.V112.11.3120.3120

Wang, M., Yang, Y., Xu, J., Bai, W., Ren, X., and Wu, H. (2018). CircRNAs as biomarkers of cancer: a meta-analysis. BMC Cancer 18:303. doi: 10.1186/s12885-018-4213-0

Wu, D. M., Wen, X., Han, X. R., Wang, S., Wang, Y. J., Shen, M., et al. (2018). Role of Circular RNA DLEU2 in human acute myeloid leukemia. Mol. Cell. Biol. 38:e00259-18. doi: 10.1128/MCB.00259-18

Wu, W., Wu, Z., Xia, Y., Qin, S., Li, Y., Wu, J., et al. (2019). Downregulation of circ_0132266 in chronic lymphocytic leukemia promoted cell viability through miR-337-3p/PML axis. Aging 11, 3561-3573. doi: 10.18632/aging.101997

Wu, Z., Sun, H., Liu, W., Zhu, H., Fu, J., Yang, C., et al. (2020). CircRPL15: a plasma circular RNA as novel oncogenic driver to promote progression of chronic lymphocytic leukemia. Leukemia 34, 919-923. doi: 10.1038/s41375-019-0594-6

Xia, L., Wu, L., Bao, J., Li, Q., Chen, X., Xia, H., et al. (2018). Circular RNA circ$\mathrm{CBFB}$ promotes proliferation and inhibits apoptosis in chronic lymphocytic leukemia through regulating miR-607/FZD3/Wnt//-catenin pathway. Biochem. Biophys. Res. Commun. 503, 385-390. doi: 10.1016/j.bbrc.2018.06.045

Yi, Y. Y., Yi, J., Zhu, X., Zhang, J., Zhou, J., Tang, X., et al. (2019). Circular RNA of vimentin expression as a valuable predictor for acute myeloid leukemia development and prognosis. J. Cell. Physyiol. 234, 3711-3719. doi: $10.1002 /$ jcp. 27145

Yuan, D. M., Ma, J., and Fang, W. B. (2019). Identification of non-coding RNA regulatory networks in pediatric acute myeloid leukemia reveals circ0004136 could promote cell proliferation by sponging miR-142. Eur. Rev. Med. Pharmacol.Sci. 23, 9251-9258. doi: 10.26355/eurrev_201911_19417

Zhang, L., and Shay, J. W. (2017). Multiple roles of APC and its therapeutic implications in colorectal cancer. J. Natl. Cancer Inst. 109:djw332. doi: 10.1093/jnci/djw332

Zhang, P., Wu, W., Chen, Q., and Chen, M. (2019). Non-coding RNAs and their integrated networks. J. Integr. Bioinform. 16:20190027. doi: 10.1515/jib-2019-0027

Zhou, H., and $\mathrm{Xu}, \mathrm{R}$. (2015). Leukemia stem cells: the root of chronic myeloid leukemia. Protein Cell 6, 403-412. doi: 10.1007/s13238-015-0143-7

Zhou, J., Zhou, L. Y., Tang, X., Zhang, J., Zhai, L. L., and Yi, Y. (2019). Circ-Foxo3 is positively associated with the Foxo3 gene and leads to better prognosis of acute myeloid leukemia patients. BMC Cancer 19:930. doi: $10.1186 / \mathrm{s} 12885-019-5967-8$

Conflict of Interest: The authors declare that the research was conducted in the absence of any commercial or financial relationships that could be construed as a potential conflict of interest.

Copyright $\odot 2020$ Perez de Acha, Rossi and Gorospe. This is an open-access article distributed under the terms of the Creative Commons Attribution License (CC BY).

The use, distribution or reproduction in other forums is permitted, provided the original author(s) and the copyright owner(s) are credited and that the original publication in this journal is cited, in accordance with accepted academic practice. No use, distribution or reproduction is permitted which does not comply with these terms. 